\section{Methotrexate in management of Morbidly Adherent Placenta at Latifa Hospital, DHA, Dubai, UAE.: Case report}

\author{
Atif BE Fazari ${ }^{1,2 *}$, Maria Eugenia Ramirez Aristondo ${ }^{1}$, Faiqa \\ Azim¹, Basma Abdo AlMaamari' and Rasha Eltayeb ${ }^{1}$ \\ 1'Department of Obstetrics \& Gynecology, Latifa Hospital, DHA, Dubai, UAE \\ ${ }^{2}$ Faculty of Medicine, University of Medical Sciences \& Technology, Khartoum, Sudan
}

\section{Abstract}

Morbidly adherent placenta (MAP) includes the spectrum of placenta accreta, increta, and percreta. It is a major cause of obstetric hemorrhage. Caesarean section is main risk factor for MAP. Ultrasound scan is highly sensitive method for MAP diagnosis and sometime Magnetic resonance image is of choice. Early diagnosis timed elective planned intervention after preparation under skillful multidisciplinary team improve the outcome and minimize the morbidity. Caesarean hysterectomy, major arteries ligation, arteries embolization and leave the placenta in-situ all are choices of management. Use of Methotrexate for the placenta in-situ in MAP is still debatable. We present a case of MAP in which placenta left in- situ followed by multiple Methotrexate injection during postpartum with good outcome and acceptability.

\section{More Information}

*Address for Correspondence: Dr. Atif Bashir Eltayeb Fazari, Consultant, Gynecologist and Obstetrician, Department of Obstetrics and Gynecology, Latifa Hospital, DHA, Dubai, UAE, Faculty of Medicine, University of Medical Sciences and Technology, Khartoum, Sudan, Tel: 00971528645050; Email: atiffazari@hotmail.co.uk

Submitted: 02 July 2019

Approved: 11 July 2019

Published: 12 July 2019

How to cite this article: Fazari ABE, Aristondo MER, Azim F, Al Maamari BA, Eltayeb R. Methotrexate in management of Morbidly Adherent Placenta at Latifa Hospital, DHA, Dubai, UAE.: Case report. Clin J Obstet Gynaecol. 2019; 2: 090-094.

\section{DOI: 10.29328/journal.cjog.1001027}

Copyright: (C) 2019 Fazari ABE, et al. This is an open access article distributed under the Creative Commons Attribution License, which permits unrestricted use, distribution, and reproduction in any medium, provided the original work is properly cited

Keywords: Morbidly adherent placenta; Placenta in-situ; Methotrexate

A) Check for updates

\section{Introduction}

Morbidly adherent placenta (MAP) includes the spectrum of placenta accreta, increta, and percreta. It is a cause of major morbidity and mortality in pregnant women. Early identification of risk factors, accurate diagnosis and treatment in accordance with the resources available are essential and may result in reduced maternal morbidity and mortality.

Myometrial tissue trauma and scarring are the main predisposing factors resulting in both placenta previa and morbidly adherent placenta. This can be caused by multiple Caesarean sections; this risk rises as the number of prior Caesarean sections increases. Placenta accreta spectrum is not exclusively a consequence of Caesarean delivery. Other surgical trauma to the integrity of the uterine endometrium and/or superficial myometrium, such as dilatation and curettage and other surgical injuries to the myometrium: manual removal of the placenta, postpartum endometritis or myomectomy, has been associated with accreta placentation in subsequent pregnancies, as well as, assisted reproductive technology and maternal smoking.

Accurate diagnosis antenatally allows for appropriate planning of delivery to minimize morbidity. Ultrasound scan and Doppler are first-line investigations with high sensitivity. Transabdominal ultrasound is noninvasive; however, it may be difficult to visualize the lower uterine segment. Transvaginal ultrasound and color Doppler may improve the diagnostic accuracy especially in patients with placenta previa as it allows detailed visualization. Recent studies looking at the use of MRI have not demonstrated superiority of this modality over transvaginal ultrasound [1]. Anyhow the MAP diagnosis can be difficult.

The surgical management choices may be considered according to available expertise, geographical and individual circumstances. Conservative approaches for women who wish to preserve their fertility aim to avoid hysterectomy. Some conservative treatments for uterus preservation include expectant management (wait and see), uterine artery embolization, Methotrexate therapy and uterus preserving surgeries (such as uterine internal/iliac artery ligation and balloon tamponade) [2].

Methotrexate has been used for many indications, including the treatment of Rheumatoid arthritis and other 
autoimmune disorders, the treatment of ectopic pregnancy, and the treatment of multiple cancers including placental site tumors and now cases of morbid adherent placenta.

It has been hypothesized that methotrexate acts by inducing placental necrosis and expediting a more rapid involution of placenta. This contradicts the belief that it acts only on rapidly dividing cells, given that trophoblastic proliferation does not occur at term. Thus, there is controversy regarding the effectiveness as an adjuvant treatment. Also, there is lack of consensus regarding optimum dosing, frequency or route of administration [3].

Publications report successful cases of conservative treatment of placenta accreta with methotrexate. To date, this medical treatment has been reported in isolated cases. The conservative method was first described by Arul Kumaran et al. in 1986 in which systemic Methotrexate was administered postnatally, and the placental mass was expelled 11 days postnatally. Since then number of cases treated conservatively have been reported [4].

The dose and method of methotrexate administration varied among different studies. Among the patients who received methotrexate, administration routes included intramuscular injection 48\%; umbilical vein injection with subsequent intramuscular injection 19\%, oral administration 4\%; and iliac catheter administration with subsequent intramuscular injection $4 \%$. Treatment by these routes of administration was successful in 6 of 11 patients (55\%), 3 of 4 patients (75\%), 0 of 1 patient $(0 \%)$, and 0 of 1 patient $(0 \%)$, respectively [5].

Although conservative management of MAP appears to be successful at preventing hysterectomy in most cases but there is always a potential for morbidity. Methotrexate use can be associated with a variety of adverse effects over a wide range of severity; The side effect profile of Methotrexate varies markedly according to dose. Regimens containing Methotrexate are classified as high $(\geq 500 \mathrm{mg} / \mathrm{m} 2)$, intermediate (between $50-500 \mathrm{mg} / \mathrm{m} 2)$, or low-dose $(<50 \mathrm{mg} / \mathrm{m} 2)$. The most commonly observed side effects at intermediate doses are rarely life threatening, this includes gastrointestinal problems such as nausea, stomatitis, soreness of mouth, fever, fatigue and headache. Most commonly reported complication in morbid adherent placenta cases managed with Methotrexate is hemorrhage (post-operative Methotrexate was associated the blood loss of 1000-2000 mL) and fever which is mostly secondary to endomyometritis or florid sepsis. Fever may also represent an inflammatory response to tissue necrosis in the absence of any infectious source. Infectious morbidity can be reduced by use of prophylactic broad-spectrum antibiotic therapy.

There is also a concern that use of Methotrexate in a lactating mother could potentially expose her neonate to harmful effects of this medication. A case report found the relative infant dose of Methotrexate to be $0.11 \%$. Methotrexate does transfer into breast milk, although the levels detected were very low. However, caution should still be used in counseling mothers regarding breastfeeding with this toxic drug. Because Methotrexate is very water soluble, it may be due to rapid renal clearance of water postnatally. Based on the drug's poor lipid solubility, $98 \%$ of Methotrexate is polar or lipid insoluble at physiological pH. This could account for its minimal secretion into breast milk. Although this is only a single case report of a rather high dose, more such studies are warranted to determine whether kinetics of transfer is similar for all women [6].

We present case report of bad morbid adherent placenta managed successfully by repeated doses of Methotrexate therapy.

\section{Case Report}

A 34-year-old woman, Gravida 4 Para 2 Abortion 1, referred to the antenatal clinic at 31 weeks of gestation as a case of placenta previa/ accreta. The patient was asymptomatic, there was no history of bleeding per vagina. There was no history of medical illness. Her surgical history include appendectomy. Her obstetrical history was marked by two previous elective Caesarean sections and one spontaneous first trimester miscarriage, medically managed without complication. At presentation, she was pale. Systemic examination revealed normal findings. Obstetrics proved fundal level almost corresponds to date, longitudinal lie and cephalic presentation with average amount of liquor and normal fetal heart sound. Ultrasound scan was performed, showed that the placenta covers the uterine cervix. Loss of retro-placental lucency was noted with rich retro-placental vascularity associated with multiple venous lakes. Patient was admitted to the hospital, received betamethasone aiming fetal lungs maturation and kept under observation.

All investigations were performed as per protocol and detailed Ultrasound scan was done which shows features of morbid adherent placenta (Figure 1). Complete blood picture showed iron deficiency anemia, she received 2 units of red blood cells. The patient and her family well counseled and explained in detail about the procedures, morbid adherent placenta protocol and hospital guidance (Table 1).

She accepted all options and she preferred if possible,
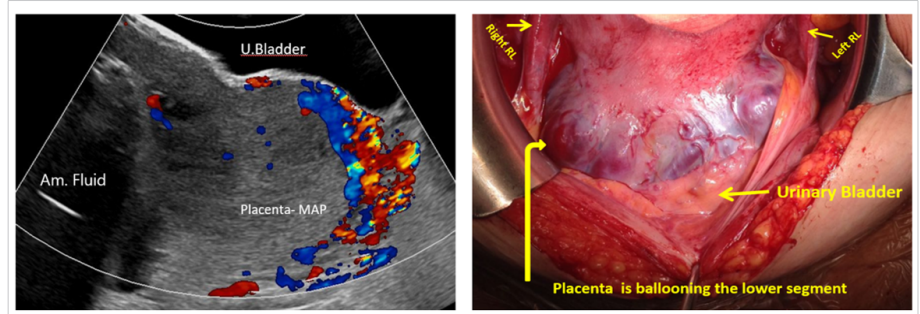

Figure 1: Shows the MAP with high vascularity with whole uterine wall invasion by placental tissues with intraoperative findings- (RL: round ligament). 
Table 1: Shows the guidance in case management of placenta in- situ.

- $\quad$ Counseling and education

- Hospital stay and intensive care admission (on need)

- Antibiotics

- Thromboprophylaxis.

- Early stents/ Ureteric catheters removal.

- Wound care.

- Methotrexate side effects

- Lactation issues with Methotrexate.

- Infection profiles.

- Serial Blood and organs tests.

- Serial ßhCG level

- Serial USS

- Follow up and clinic appointment.

- Hospital accessibility and easy staff contact

- Risk of hysterectomy

to reserve uterus and has no objection for Methotrexate injections with agreement of lactation issues. Three weeks later at 34 weeks the patient underwent the surgery and the following procedures were done:

1. Cystoscopy with bilateral ureteric stenting.

2. Classical Caesarian section with outcome of male baby $2.630 \mathrm{~kg}$.

3. Placenta kept in-situ.

4. Bilateral Iliac arteries ligation.

She tolerated the surgery well no intraoperative nor postoperative complications noted. She received:

1. Antibiotics for 10 days.

2. Thromboprophylaxis.

3. Started breastfeeding the first 10 days post-surgery.

4. Received THREE doses of methotrexate injection according to the body surface area calculation staring from the first week then third week and fifth week postpartum. (In two weeks interval)

5. Had serial follow up of beta hCG and ultrasound scan.

6. Continue breast feeding between the Methotrexate doses under neonatology department follow up.

Patient had been kept 3 weeks postpartum for close monitoring. No bleeding or infection seen during inpatient period. Healthy clean wound at the time of discharge. She had been kept under interval of two weeks follow up with education for any symptoms and signs of infection along with serial USS study for the placenta measurement and fluid volume collection which was observed inside uterine cavity during the placental collapse (Table 2) (Figure 2). With USS the beta HCG also measured and seen in table 3, decreasing significantly. On week 26 post-surgery patient expelled the placenta completely sent for histopathology (Figure 3). The uterus well contracted, no bleeding. Estimated blood Loss $200 \mathrm{ml}$.

Histopathology Report showed products of conception composed of necrotic placental tissue and umbilical cord.

\begin{tabular}{|c|c|c|c|}
\hline USS & Week & Tissue measurement & Volume \\
\hline $\begin{array}{l}\text { 1st Ultrasound } \\
\quad\left(2^{\text {nd }} w k\right)\end{array}$ & $2^{\text {nd }}$ week & $\begin{array}{c}\text { Placental measuring } 14.0 \times 8.3 \times 13.5 \\
\text { cm along its maximal TS, AP and CC } \\
\text { diameters respectively and No free } \\
\text { fluid collections. }\end{array}$ & $815.7 \mathrm{ml}$ \\
\hline 2nd Ultrasound & $4^{\text {th }}$ week & $\begin{array}{l}\text { Its anterior portion is seen measuring } \\
11.3 \times 9.3 \times 11.6 \mathrm{~cm} \text {, while its } \\
\text { posterior portion measures } 11.4 \times 3 \\
\times 9.6 \mathrm{~cm} \text { with estimated volume of } \\
172 \mathrm{~cm}^{3} .\end{array}$ & $640 \mathrm{ml}$ \\
\hline 3rd Ultrasound & $6^{\text {th }}$ week & $\begin{array}{c}\text { Placenta seen in lower endometrial } \\
\text { cavity measuring } 1.0 \times 10.4 \times 9.3 \mathrm{~cm} \text {. } \\
\text { No free fluid is seen }\end{array}$ & $515 \mathrm{ml}$ \\
\hline 4th Ultrasound & $8^{\text {th }}$ week & $\begin{array}{c}\text { Anterior portion } \\
\text { measures } 8.7 \times 6.2 \times 5.5 \mathrm{~cm} \\
\text { Posterior portion measures } 7.6 \times 2.5 \\
\times 5.2 \mathrm{~cm}\end{array}$ & $\begin{array}{c}154.4 \mathrm{ml} \\
\text { anterior part } \\
\text { and } 51.8 \mathrm{ml} \\
\text { posterior part. }\end{array}$ \\
\hline
\end{tabular}

\begin{tabular}{|c|c|}
\hline Table 3: Follow up of Beta HCG result. & \\
\hline Time & Result \\
\hline 1st day post-surgery & $5797 \mathrm{mlU} / \mathrm{mL}$ \\
\hline 1 week & $3546 \mathrm{mlU} / \mathrm{mL}$ \\
\hline 2 weeks & $2272 \mathrm{mlU} / \mathrm{mL}$ \\
\hline 3 weeks & $899 \mathrm{mlU} / \mathrm{Ml}$ \\
\hline 4 weeks & $100 \mathrm{mlU} / \mathrm{mL}$ \\
\hline 5 weeks & $<5 \mathrm{mlU} / \mathrm{mL}$ \\
\hline 6 weeks & $<5 \mathrm{mlU} / \mathrm{mL}$ \\
\hline
\end{tabular}

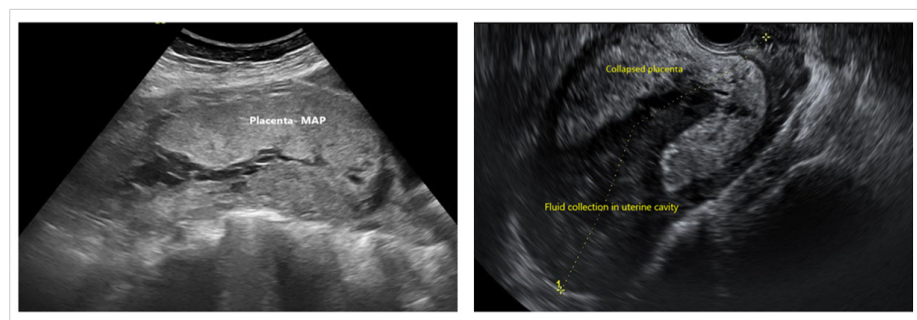

Figure 2: Shows the process of placental collapse.
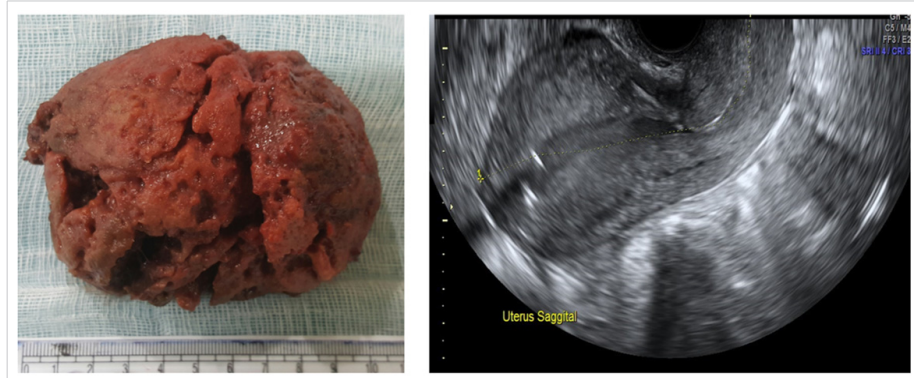

Figure 3: A photograph of the placental tissue after expulsion and Trans- vaginal scan image shows empty uterine cavity

The patient kept for one day in hospital for observation and discharged in good condition.

\section{Discussion}

This case report represented a successful conservative management of MAP with Methotrexate injection. Sentilhis et al. reviewed conservative management in 167 cases of placenta accreta/percreta. The failure rate of conservative management was $22 \%$ which required hysterectomy, either primary or delayed, mostly for severe hemorrhage. Conservative treatment was successful for 131 women (78.4\%). Out of the 
remaining 36 women, 18 had primary hysterectomy and 18 had delayed hysterectomy (10.8\% each). Severe maternal morbidity occurred in 10 cases $(6.0 \%)$. One mortality was reported due to myelosuppression and nephrotoxicity related to intraumbilical methotrexate administration. Placental resorption occurred spontaneously in 87 of 116 cases (75\%), with an average interval from delivery of 13.5 weeks (range 4-60 weeks).

Treating an abnormally adherent placenta conservatively must be weighed against the risk of several associated complications, being the most significant the bleeding or secondary postpartum hemorrhage.

In this presented case, the patient was initially diagnosed with placenta percreta using ultrasound. Classical Caesarean section was performed and the morbid adherent placenta left in situ She was then monitored with serial ultrasound after looking for devascularisation of the placental bed which ends up in a complete expulsion of the placenta 26 weeks post-surgery. Ultrasound is an effective imaging modality for diagnosing and monitoring placental invasion. Additionally, HCG was successfully used as a method of monitoring placental vascular activity even though there are several documented cases of persistent vascularity despite undetectable HCG levels [7].

In a retrospective analysis, Chunhua Zhang et al., studied 54 cases who were diagnosed of placenta increta. Patients were treated with methotrexate via two different routes of administration: intravenously and with local multipoint injection under ultrasound guidance. They found that Local multi-point methotrexate injection under ultrasound guidance is a better alternative for patients with placenta increta, especially for preserving fertility [8].

The purpose of conservative management of abnormally invasive placentation is to allow the placenta time to devitalize and to make the removal less difficult [9]. Following a systematic approach, the main complications were evaded including surgical removal of the invasive placental tissue, ultimately preserving the uterus and performing merely a classical Caesarean section. If delivery and removal of the placenta had been attempted at time of diagnosis, bleeding from the placental bed may well have been more difficult to control leading to a hysterectomy.

Both UK and American guidelines are clear that these women must be managed in a center capable of rapid blood transfusions, with emergency operating theatres, experienced multidisciplinary surgeons, and intensive care [9]. This was reinforced by the management and outcome of this case, as this patient received blood transfusion prior the surgery, ureteric stent was inserted by urologist and well managed by expert multidiciplinary team.

Other significant risks during the observation period in- clude infection, ongoing bleeding, and dissaminated coagulopathy, risks that were decreased by a protocol of management which include implementation of prophylactic antibiotherapy and thromboprophylaxis. This stepwise approach can be easily implemented leading to prevention of secondary surgical interventions [10].

Despite these risks, conservative management will continue to be a desirable option for women wanting to preserve their fertility, avoiding hysterectomy and establishing a low rate of severe maternal morbidity in centers with adequate equipment and resources as the one which this case was performed.

Regarding methotrexate, to date, this type of medication has only been reported in isolated cases, but it has been suggested as an adjuvant treatment during the observation period in order to target folate metabolism in the rapidly growing tissues of the trophoblast. There is currently no clear evidence as to the benefit of methotrexate in conservative management of placenta accreta after delivery. Which is essential to emphasize, it is the lapse of time given for breastfeeding; this therapy was not started immediately after the surgery in that way providing time for breastfeeding and milk expression.

Our result is therefore reassuring and suggest that successful conservative treatment for morbid adherent placenta does not appear to compromise the patients' subsequent fertility or obstetric outcome, but that the risk of morbid adherent placenta recurrence during future deliveries is high. Further studies including multiple centers and uniform diagnostic criteria are needed to identify optimal management strategies for this increasingly common, morbid condition.

\section{Conclusion}

Classical Caesarean section and conservative treatment for morbid adherent placenta is accepted modality in such cases. Counseling and case education are crucial concerning the methotrexate, sepsis and antibiotics usage. Multidisciplinary team of specialties is skeleton of patient care. Lack of evidence base is one of the constrain to accredit Methotrexate injection in MAP management; almost the available evidence is from cases reports and yet no high level of evidence available like Randomize control study.

\section{Acknowledgement}

Special thanks to Thanks for Radiology, Anesthesia, laboratory and nursing departments at Latifa hospital and appreciation for their input in case care.

\section{Ethical approval}

Informed consent obtained from the case herself with agreement of Obstetrics\& Gynecology department for publication with confidentiality. 


\section{References}

1. Jauniaux ERM, Alfirevic Z, Bhide AG, Belfort MA, Burton GJ, et al. Placenta Praevia and Placenta Accreta: Diagnosis and Management. Green-top Guideline No. 27a. BJOG. 2019; 126: e1-e48. PubMed.: https://www.ncbi.nlm.nih.gov/pubmed/30260097

2. Babaei MR, Oveysi Kian M, Naderi Z, Khodaverdi S, Raoofi Z, et al Methotrexate infusion followed by uterine artery embolisation for the management of placental adhesive disorders: a case series. Clin Radiol. 2019; 74: 378-383. PubMed.: https://www.ncbi.nlm.nih.gov/ pubmed/30755315

3. Khan M. Sachdeva R, Arora R, Bhasin S. Conservative management of morbidly adherant placenta-A case report and review of literature. Placenta. 2013; 34: 963-966. PubMed.: https://www.ncbi.nlm.nih.gov/ pubmed/23937959

4. Arulkumaran $\mathrm{S}, \mathrm{Ng} \mathrm{CS}$, Ingemarsson I, Ratnam SS. Medical treatment of placenta accreta with methotrexate. Acta Obstet Gynecologica Scand. 1986; 65: 285-286. PubMed: https://www.ncbi.nlm.nih.gov/ pubmed/3739639

5. Matsuzaki S, Yoshino K, Endo M, Kakigano A, Takiuchi T, et al.
Conservative management of placenta percreta. Int J Obstet Gynecol. 2017.

6. Baker T, Datta P, Rewers-Felkins K, Thomas W. Breastfeeding Medicine. 2018.

7. Timmermans $\mathrm{S}$, van Hof AC, Duvekot JJ. Conservative management of abnormally invasive placentation. Obstet Gynecol Surv. 2007; 62: 529539. PubMed.: https://www.ncbi.nlm.nih.gov/pubmed/17634154

8. Matsubara S, Takahashi H, Usui R. Letter to 'Retrospective analysis: Conservative treatment of placenta increta with methotrexate': Some clarifications. J Obstet Gynaecol Res. 2018; 44: 1499-1500. PubMed.: https://www.ncbi.nlm.nih.gov/pubmed/29932489

9. MacGibbon A, lus YM. Conservative Management of Abnormally Invasive Placenta Previa after Midtrimester Foetal Demise. Case Rep Obstet Gynecol. 2018; 7478437. PubMed.: https://www.ncbi.nlm.nih. gov/pubmed/30405926

10. Chaudhari HK, Shah PK, D'Souza N. Morbidly Adherent Placenta: Its Management and Maternal and Perinatal Outcome. J Obstet Gynaecol India. 2016; 67: 42-47. PubMed.: https://www.ncbi.nlm.nih.gov/ pubmed/28242967 